\title{
Sub-Nanometer Displacement Sensor Based on Coupling of Balanced Loss and Gain Cavities
}

\author{
Yuntuan FANG* and Xiaoxue LI \\ School of Computer Science and Telecommunication Engineering, Jiangsu University, Zhenjiang 212013, China \\ ${ }^{*}$ Corresponding author: Yuntuan FANG \\ E-mail: fang_yt1965@sina.com
}

\begin{abstract}
Sub-nanometer displacement measurement is still a challenge in the current sensor field. In this study, a new type of displacement sensor is designed which is based on the coupling effect of two balanced gain and loss resonators. The optical properties of the sensor have been studied through the coupled mode theory and scatter matrix. The pole effect in the coupling system can be used to measure the sub-nanometer displacement. The resolution of the sensor can reach $0.001 \mathrm{~nm}$ over a dynamic range of $20 \mathrm{~nm}$. The sensor has the highest sensitivity within the range of one nanometer. The environmental disturbance and structure parameter perturbation have been demonstrated to make trivial effect on the sensor performance.
\end{abstract}

Keywords: Displacement sensor; PT-symmetry; pole

Citation: Yuntuan FANG and Xiaoxue LI, "Sub-Nanometer Displacement Sensor Based on Coupling of Balanced Loss and Gain Cavities," Photonic Sensors, 2019, 9(3): 259-267.

\section{Introduction}

Precision measurement is one of the key technologies in the modern industry and a sign of technology and industrial competitive power of countries. The precise displacement determination involves all aspects of manufacturing system engineering, such as the atomic force probes $[1,2]$, the accelerometers [3], the nanopositioning equipment in machine processing[4], the scanning stages [5], the confocal laser scanning microscope [6], the structural health monitoring [7], the landslide monitoring [8], the compliant microgripper [9], and the compliant micro-positioning platform [10]. The optical displacement sensor is widely used in the field of precise and ultraprecise displacement measurement due to advantages of non-contact process, high precision, and broadband [11-13]. In recent years, laser interferometers and grating interferometers have become the main representations of the optical displacement sensor [12]. Laser interferometers are commonly used to achieve high-precision displacement sensing with independence of high-precision manufacturing process $[5,13]$. However, laser interferometers are subject to environment disturbances such as temperature, pressure, humidity variations, and even vibrations. Compared with other sensor technologies, optical-grating sensors can provide relatively large range and high precision. However, the sensing precision depends on the manufacturing precision of grating lines. Thus, the sensing accuracy is determined by the definition and homogeneity of the grating line edges. Furthermore, if the sensor relies strictly on the machining precision of lithographic techniques, it is difficult to obtain nanometer

Received: 26 September 2018 / Revised: 26 October 2018

(C) The Author(s) 2018. This article is published with open access at Springerlink.com

DOI: $10.1007 / \mathrm{s} 13320-018-0526-5$

Article type: Regular 
resolution owing to the fundamental diffraction limit of the optical scanning method $[14,15]$. In the current nanotechnology age, displacement sensors with nanometer accuracy and resolution are crucial for precision positioning and machining systems. To overcome the limitation of optical-grating sensors, the time grating approach has been proposed to design the displacement sensor in which the space grating is replaced by time grating. The sensing precision reaches $\pm 200 \mathrm{~nm}$ and the resolution reaches $1 \mathrm{~nm}$ within $200 \mathrm{~mm}$. However, in the nanopositioning system and nanofabrication, such as the scanning tunneling microscope [16] and the atomic force microscope (AFM) [17], they need the sub-nanometer precision. In the scanning tunneling microscope, the distance between probe and specimen of scanning tunneling microscope is less than $1 \mathrm{~nm}$. A slight variation in the distance will affect the sensing results. Therefore, the distance between probe and specimen must be strictly controlled. The AFM is proposed to measure the photo-induced nanometer-scale surface displacement in solids, and the variation of displacement with time is much less than $1 \mathrm{~nm}$. It is required to design the displacement sensor with sub-nanometer resolution or nanometer resolution. It is still a challenge to achieve the devices.

Recently, parity-time (PT)-symmetry systems have been studied in optical structures. The PT-symmetry systems have a unique exceptional point (EP) effect and pole effect [18-24]. These interesting physics have opened a new window in designing some new kinds of photonic device, such as lasing and anti-lasing transformer [19], sensor [20, 21], and optical isolator [22]. In this paper, a layered resonator coupling system with quasi-PT-symmetry configuration is designed. Such a structure leads to an isolated pole with a large transmittance. Because the pole mode is resulted from the mode coupling through the air cavity, the transmittance of the structure is much sensitive to the thickness of the air layer. Such a mechanism can be used to design a new type of displacement sensor. It detects displacement by the transmittance at the pole. After the light power is transformed into electric current through photoelectric sensors, the results are read directly through the change of light current. The numerical results show that the sensor takes a great advantage over the traditional displacement sensors.

\section{Models and methods}

As shown in Fig. 1, two cavities formed by two metal layers (the yellow layers in the figure) and medium layer ( $\mathrm{G}$ or $\mathrm{L}$ ) are fixed on the fixed base and movable object, respectively. The air layer is between the two resonators. Layers $\mathrm{G}$ and $\mathrm{L}$ are made of gain and loss medium, respectively. The refraction indexes and thicknesses of all layers are denoted as $n_{\mathrm{M}}, n_{\mathrm{G}}, n_{\mathrm{L}}, n_{\mathrm{A}}$, and $d_{\mathrm{M}}, d_{\mathrm{G}}=d_{\mathrm{L}}, d_{\mathrm{A}}$, respectively. Based on the Drude model, $\mathrm{n}_{\mathrm{M}}$ is given by [25]

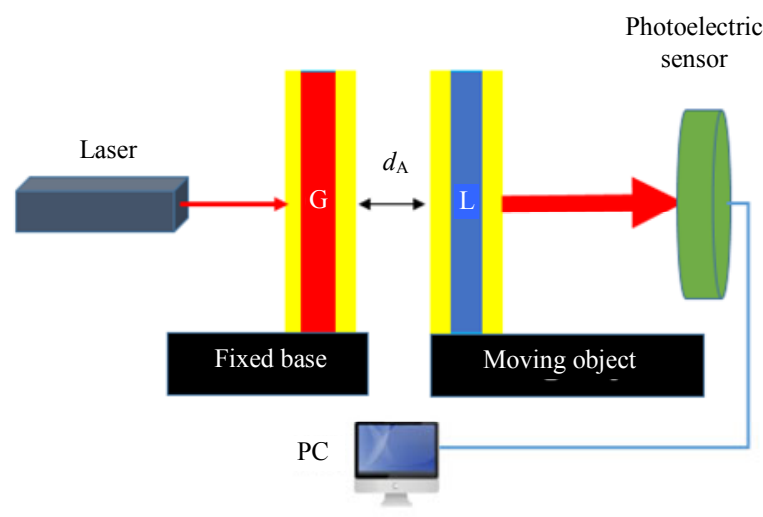

Fig. 1 Schematic of the displacement sensor.

$$
n_{\mathrm{M}}=\sqrt{1-\frac{\omega_{\mathrm{ep}}^{2}}{\omega^{2}}}
$$

where $\omega$ is the frequency of incident wave, and $\omega_{\mathrm{ep}}$ is the frequency of electronic plasma that equals to $1.2 \times 10^{16} \mathrm{~s}^{-1}$. For $\omega<\omega_{\mathrm{ep}}, n_{\mathrm{M}}$ is an imaginary number. On this case, a reflection occurs when the incident wave meets the mental layer, and the evanescent fields will enter into layers $G, L$, and A. The electromagnetic waves in layers $\mathrm{A}, \mathrm{G}$, and $\mathrm{L}$ will be reflected on their two interfaces. When the 
incident wavelength satisfies the condition of resonance, layers $\mathrm{A}, \mathrm{G}$, and $\mathrm{L}$ will become three resonators. The gain layer $G$ could be achieved through the material based on thick InGaAsP multiple quantum wells [19]. The loss layer $C$ could be achieved through the absorbing $\mathrm{Cr} / \mathrm{Ge}$ material. In the PT symmetry structure, the distribution of the refractive index should be an even function for the real part, and the imaginary part should be an odd function. The refraction indexes of layers $\mathrm{G}$ and $\mathrm{L}$ can be adjusted to be $n_{\mathrm{G}}=n-\mathrm{i} \tau$ and $n_{\mathrm{L}}=n+\mathrm{i} \tau$, respectively, to satisfy the PT-symmetry condition. The value of $\tau$ denotes the intensity of gain or loss. As is known, the optical PT-symmetry condition requires that the refraction index distribution is a conjugate symmetric function, i.e., $n(\mathbf{r})=n^{*}(-\mathbf{r})$ [18]. However, $n_{\mathrm{M}}$ in our structure has an imaginary value, the condition of $n(\mathbf{r})=n^{*}(-\mathbf{r})$ in Fig. 1 is not satisfied. In this case the metal film induces an additional perturbation and the system becomes quasi-PT symmetry. Because the metal film is very thin, the perturbation is trivial. In this paper, the structure is still studied with the help of PT-symmetry theory.

There could be coupling effect among the three resonators $\mathrm{L}, \mathrm{G}$, and $\mathrm{A}$. The coupling effect can be observed from the transmission spectra. The transmission spectra can be obtained through the calculations based on the transfer matrix method [26]. The incidence angle and structure parameters are taken as: $\theta=0^{\circ}, n=3.205, \tau=0$, and $n_{\mathrm{A}}=$ 1.00027 , and $d_{\mathrm{M}}=50 \mathrm{~nm}, d_{\mathrm{G}}=d_{\mathrm{L}}=676 \mathrm{~nm}$, and $d_{\mathrm{A}}=$ $2320 \mathrm{~nm}$. These parameters have been optimized to achieve the coupled modes around $1550 \mathrm{~nm} . \tau=0$ denotes a passive structure. The transmittance spectra are plotted in Fig. 2. In the Figure, the left two close peaks correspond to the coupled modes of the resonators $\mathrm{L}$ and $\mathrm{G}$. The right isolated peak corresponds to the resonance mode of layer A. All the peaks have the unit peak values. In general, the intense coupling occurs among the modes with small intervals. Because the right peak is far away from the left peaks, the mode of the resonator $\mathrm{A}$ is not coupled with the modes of the resonators $\mathrm{G}$ and $\mathrm{L}$, but it will influence the coupling coefficient of resonators $\mathrm{G}$ and $\mathrm{L}$ through the change of thickness. In the following, the coupling effect of the resonators $\mathrm{G}$ and $\mathrm{L}$ with $\tau \neq 0$ will be studied.

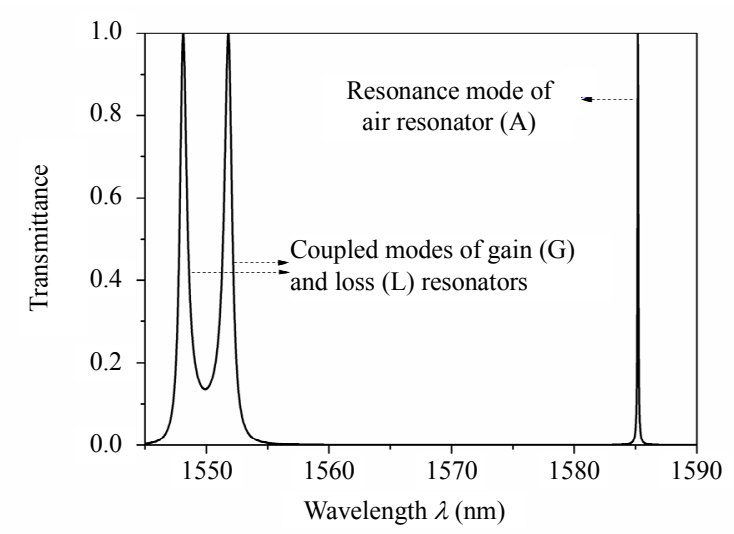

Fig. 2 Transmission spectra of the structure in Fig. 1 with $\tau=0$.

The modal field evolution in the layers $\mathrm{G}$ and $\mathrm{L}$ can be analyzed through the coupled mode theory. The equations can be given in the following equation [22]:

$$
\left\{\begin{array}{l}
d a_{1} / d t=-\mathrm{i}\left(\omega_{0}+\mathrm{i} g\right) a_{1}-\mathrm{i} \kappa a_{2} \\
d a_{2} / d t=-\mathrm{i}\left(\omega_{0}+\mathrm{i} \gamma\right) a_{2}-\mathrm{i} \kappa a_{1}
\end{array}\right.
$$

where $a_{1}$ and $a_{2}$ are the field amplitudes of layers $\mathrm{G}$ and $\mathrm{L}$, respectively, and $\omega_{0}$ is the resonance frequency of the two resonators with the same thickness. $g$ and $\gamma$ are the gain and loss coefficients of the two cavities, respectively, and their values are dependent on the value of $\tau . k$ is the coupling efficient of the two resonators. Assuming the system has the form of $\mathrm{e}^{-\mathrm{i} \omega t}$, where $\omega$ is the eigenfrequency, then it will leads to

$$
\frac{d}{d t}\left[\begin{array}{l}
a_{1} \\
a_{2}
\end{array}\right]=-\mathrm{i} \omega\left[\begin{array}{l}
a_{1} \\
a_{2}
\end{array}\right] .
$$

Combining (2) and (3) leads to the eigen matrix equation as follows:

$$
\left[\begin{array}{cc}
\left(\omega_{0}+\mathrm{i} g\right) & \kappa \\
\kappa & \left(\omega_{0}+\mathrm{i} \gamma\right)
\end{array}\right]\left[\begin{array}{l}
a_{1} \\
a_{2}
\end{array}\right]=\omega\left[\begin{array}{l}
a_{1} \\
a_{2}
\end{array}\right] .
$$

In the PT-symmetry system, it satisfies $\gamma=-g$. Through solving the algebraic equation, the following equation is obtained as follows: 


$$
\omega=\omega_{0} \pm \sqrt{\kappa^{2}-g^{2}} .
$$

Equation (5) determines the system state. If $k>g$, $\omega$ has two real values denoting the PT-symmetry state and a mode splitting. $k=g$ results in the exceptional point at which the two eigenstates are just coalesced. If $k<g, \omega$ has two complex values denoting the broken PT-symmetry state. In fact, the behavior of the PT-symmetry structure can be also described by the eigenvalues of the scattering matrix S. The $\mathbf{S}$ matrix describes the relation between the reflectance and incidence on the structure and is denoted as (see Appendix)

$$
\left[\begin{array}{l}
a \\
d
\end{array}\right]=\mathbf{S}\left[\begin{array}{l}
b \\
c
\end{array}\right]=\left[\begin{array}{ll}
s_{11} & s_{12} \\
s_{21} & s_{22}
\end{array}\right]\left[\begin{array}{l}
b \\
c
\end{array}\right]
$$

where $a$ and $d$ denote the reflectances from the incidence and output spaces, and $b$ and $c$ denote the incidences from the incidence and output spaces. The eigenvalues of the $\mathbf{S}$ matrix either form pairs with reciprocal moduli corresponding to the broken PT-symmetry state or become unimodular corresponding to the PT-symmetry state. In the broken PT-symmetry state, some poles or zero points may occur $[23,24]$. The pole and zero points correspond to amplification and attenuation, respectively. In the current system, on the PT-symmetry condition ( $\tau \neq 0$ ), the coupling effect of resonators $\mathrm{G}$ and $\mathrm{L}$ will lead to the pole effect. The pole effect is just the sensing basis in this study.

\section{Results and analysis}

It is clear that the values of the three peaks in Fig. 2 are all equal to unit. Keeping the structure parameters in Fig. 2 and increasing $\tau$ by steps, the transmittance spectra of the structure is calculated and plotted in Fig. 3. With an increase in $\tau$, the left two transmittance peaks will become closer and higher. When $\tau$ is up to 0.0042 , the two peaks merge into one peak at $\lambda=1550 \mathrm{~nm}$. According to (5), the merged peak has entered into the broken PT-symmetry state and has a peak value much larger than unit.

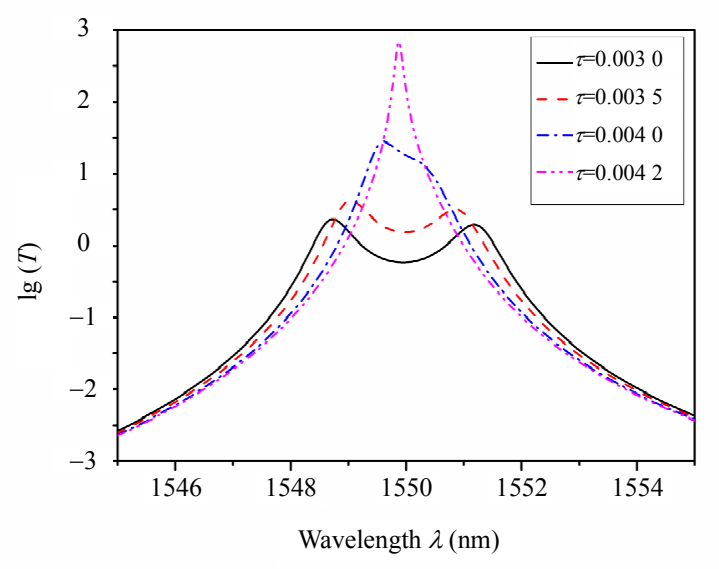

Fig. 3 Transmission spectra of the structure in Fig. 1 with different $\tau$.

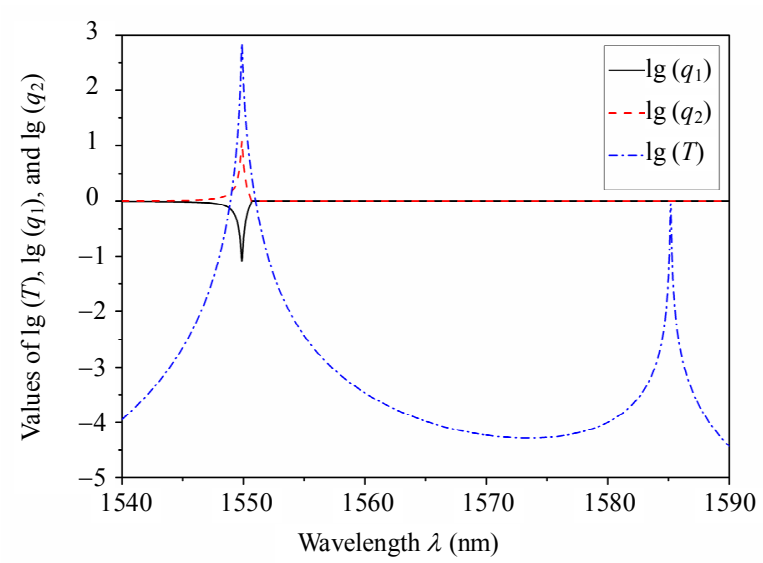

Fig. 4 Transmission spectrum and two eigen values of the scatter matrix with $\tau=0.0042$.

To make an insight into the system state, the transmission spectra in a larger range, and two eigenvalues $q_{1}$ and $q_{2}$ of the scatter matrix of the system with $\tau=0.0042$ are plotted in Fig. 4 . The right peak corresponding to layer $\mathrm{A}$ is far from the left peak, which further states that the resonator A does not join the coupling process of the resonators $G$ and L. The two reciprocal peaks of $q_{1}$ and $q_{2}$ correspond to the pole and zero points. The zero point corresponds to the coherent perfect absorption (CPA) solutions [23]. For one side incidence, only the pole effect is excited. It is clear that the left peak is just at the pole position of the two eigenvalues at $\lambda=$ $1550 \mathrm{~nm}$. Thus the maximum transmittance peak is resulted from the pole effect. Through the following study, the peak value at the pole is found to be extraordinary sensitive to the value of $d_{\mathrm{A}}$, because 
the air cavity influences the coupling efficient of the two resonators. It just offers a way to design the displacement sensor.

As schematized in Fig. 1, the resonator $\mathrm{G}$ is fixed at the base, and the resonator $\mathrm{L}$ is fixed at the moving object. Thus the change of $d_{\mathrm{A}}$ is just the displacement to be detected. It should be noted that Fig. 1 just means an experimental model. It is not a real experimental equipment. However, the experimental model can be applied to many occasions. For example, in the cutting process, the distance between the cutter and target object is very small and has to be precisely determined. Thus the resonator $\mathrm{G}$ is fixed at the target object, and the resonator $\mathrm{L}$ is fixed at the cutting. The change of $d_{\mathrm{A}}$ can determine the cutting range. The same design can be also applied to the atomic force microscope. The distance between the probe and specimen of scanning tunneling microscope will affect the measurement results. Thus the resonator $\mathrm{G}$ is fixed at the probe and the resonator $\mathrm{L}$ is fixed at the specimen. Through the changing of $d_{\mathrm{A}}$, the distance between the probe and the specimen can be determined precisely. To measure the displacement, the incidence wavelength $1550 \mathrm{~nm}$ is used to calculate the transmittance of the system on normal incidence. The changes of transmittances with $d_{\mathrm{A}}$ for different $\tau$ are plotted in Fig. 5(a). If $d_{\mathrm{A}}$ and the transmittance have one-to-one correspondence, the transmittance can be used to determine $d_{\mathrm{A}}$ and the displacement from its change. Figure 5(a) shows that the curves for $\tau=0.0042, \tau=0.00422$, and $\tau=0.00424$ all monotonically decrease with $d_{\mathrm{A}}$ increasing, but the curve of $\tau=0.00417$ has a convex shape near $d_{\mathrm{A}}=2320 \mathrm{~nm}$. Thus $\tau=0.00417$ can not be used as the sensing parameter. In this study $\tau=0.0042$ is chosen because it has larger transmittances. The slope of the curve of $\tau=0.0042$ describes how quickly the transmittance changes with $d_{\mathrm{A}}$, i.e., the sensing sensitivity. To make a quantum analysis, the sensitivity is defined as $-\Delta T /(T \Delta d)$ in which $\Delta d=0.001 \mathrm{~nm}$ is the increment of $d_{\mathrm{A}}$ for calculating $\Delta T$. The sensitivity is shown in Fig. 5(b). It is clear that the sensitivity has a maximum value of $1.27 \mathrm{~nm}^{-1}$ at $d_{\mathrm{A}}=2320.6 \mathrm{~nm}$. Such sensitivity occurs for the displacement of $0.6 \mathrm{~nm}$. To give a comparison, a displacement sensor based on the response of optical intensity should be chosen. For the intensity-modulated plastic fiber optic displacement sensor in [11], the displacement-dependent optical response $R$ is plotted as the function of displacement. The sensitivity is defined as the partial derivative of the function. In the linear region, the sensitivity is about $0.25 \mathrm{~mm}^{-1}$. In a small displacement range, the sensitivity in this study is higher than the similar sensor in [11].

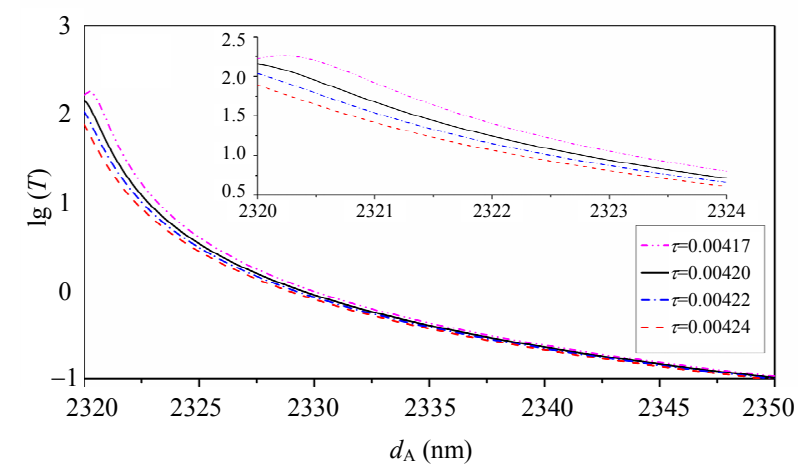

(a)

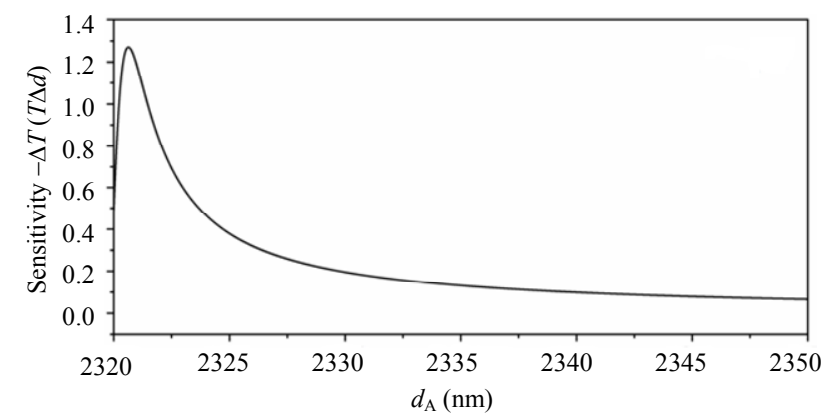

(b)

Fig. 5 Transmittance and sensitivity: (a) transmittance for different $\tau$ and (b) the sensitivity for $\tau=0.0042$.

To read the sensing results, the optical signal from the transmittance has to be transformed into the electric signal through the photoelectric sensor. The detecting resolution and range are also dependent on the photoelectric sensor. For the photoelectric sensor called variable-gain temperature-compensated avalanche photo detectors 
(APD430C), the minimum and maximum detectable optical power are the order of PW and $\mu \mathrm{W}$, which means that the ratio of the minimum and maximum detectable optical power can attain $10^{-6}$. Because the transmittance is proportional to the optical power, the minimum relative change $\Delta T / T$ could reach $10^{-6}$. To give a reliable estimation, $\Delta T / T=10^{-4}$ is regarded as the threshold value that can be detected by the photoelectric sensor. Thus an increment $\Delta d=0.001 \mathrm{~nm}$ is chosen to calculate the corresponding values of $\Delta T / T$ at different $d_{\mathrm{A}}$. The result is shown in Fig. 6 . It is seen that in the range of $d_{\mathrm{A}}$ from $2320 \mathrm{~nm}$ to $2340 \mathrm{~nm}$, the values of $\lg (\Delta T / T)$ is larger than -4 (labeled by the horizontal dash line). Thus the sensing range is $20 \mathrm{~nm}$. Because the $\Delta d$ values of $\lg (\Delta T / T)$ are based on increment $=0.001 \mathrm{~nm}$, the displacement $\Delta d=0.001 \mathrm{~nm}$ is detectable, that is, $0.001 \mathrm{~nm}$ is the sensing resolution. Therefore, a sub-nanometer displacement sensor has been achieved.

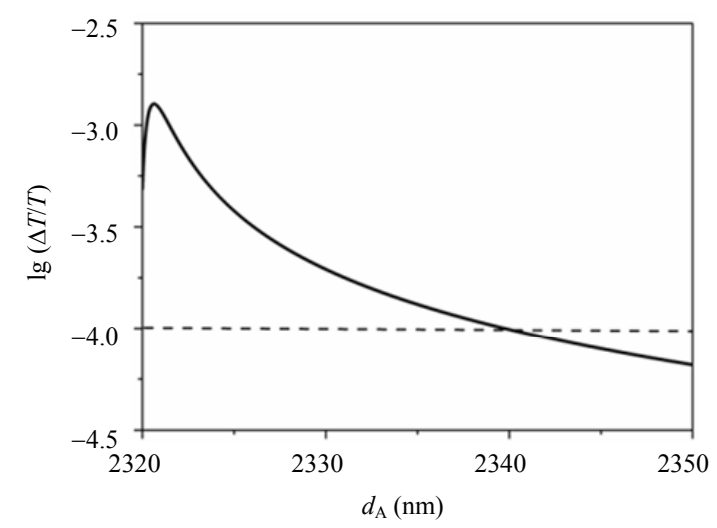

Fig. 6 Values of $\lg (\Delta T / T)$ based on the increment $\Delta d=$ $0.001 \mathrm{~nm}$.

In order to further increase the sensitivity, the thicknesses of layer $M$ are chosen as $d_{M}=49 \mathrm{~nm}$, $50 \mathrm{~nm}$, and $51 \mathrm{~nm}$ with $\tau=0.00454, \tau=0.0042$, and $\tau=0.00386$, respectively. With the same incidence wavelength $1550 \mathrm{~nm}$, we plot the transmittances of the three structures in Fig. 7. It is clear that the curves for $d_{\mathrm{M}}=49 \mathrm{~nm}$ and $d_{\mathrm{M}}=51 \mathrm{~nm}$ decrease the fastest and the slowest, respectively. It should point out that Fig. 7 is a simulation result. In a real measurement, the random disturbance of experimental condition should be considered. In this study, we mainly consider the effect of random disturbance of laser wavelength and gain coefficient $\tau$ on the sensitivity. The thicknesses of $d_{M}$ are $49 \mathrm{~nm}, \quad 50 \mathrm{~nm}$, and $51 \mathrm{~nm}$ with gain coefficient $\tau=0.00454+\Delta_{1}, \quad \tau=0.0042+\Delta_{2}, \quad$ and $\tau=0.00386+\Delta_{3}$ are chosen, respectively. The incidence wavelength is chosen as $\lambda=\left(1550+\Delta_{4}\right) \mathrm{nm} . \quad \Delta_{1}-\Delta_{3}$ are random numbers ranging from $-0.5 \times 10^{-5}$ to $0.5 \times 10^{-5}$ and $\Delta_{4}$ is a random number ranging from -0.5 to 0.5 . The sensitivities of the three structures with $d_{\mathrm{A}}$ are made twenty times. The results are plotted in Fig. 8. Each dashed line corresponds to the result with the random disturbance. The solid line is the statistical average value for the twenty calculations. The statistical value can delete the random errors owing to the random disturbance. It is clear that the average curves for $d_{\mathrm{M}}=49 \mathrm{~nm}$ and $d_{\mathrm{M}}=51 \mathrm{~nm}$ decrease the fastest and the slowest, respectively. In the range for $d_{\mathrm{A}}$ from $2320 \mathrm{~nm}$ to $2325 \mathrm{~nm}$, the sensitivity for $d_{\mathrm{M}}=49 \mathrm{~nm}$ and $d_{\mathrm{M}}=51 \mathrm{~nm}$ are the highest and the lowest, respectively. However, the three sensitivity curves are most overlapped when $d_{\mathrm{A}}$ is over $2325 \mathrm{~nm}$. The values of $\Delta T / T$ for the three structures are plotted in Fig. 9. As can be seen, the measurement ranges are almost the same from $2320 \mathrm{~nm}$ to $2340 \mathrm{~nm}$.

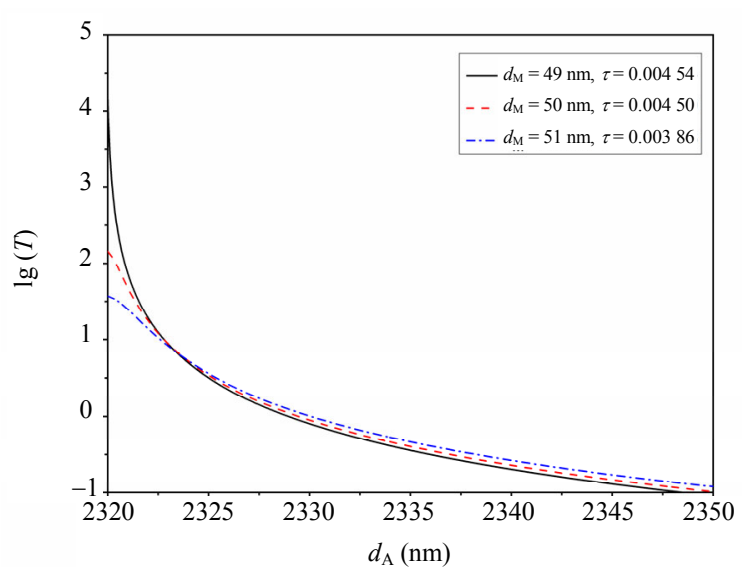

Fig. 7 Transmittances of three structures.

In practical applications, the stability and reproducibility of a sensor is very important. For the current sensor, the stability and reproducibility are related to the fault tolerance. The influence of 
parameter perturbation on the sensor performance is studied. First, $n_{\mathrm{A}}$ will be affected by the environmental disturbance such as airflow and position modification. The relative difference $\Delta T / T$ is used to describe the influence, where $\Delta T$ is the difference of the values with and without perturbation. The values of $\Delta T / T$ versus $n_{\mathrm{A}}$ for three structures are shown in Fig. 10. As can be seen, $\Delta T / T$ for $d_{\mathrm{M}}=49 \mathrm{~nm}$ and $d_{\mathrm{M}}=51 \mathrm{~nm}$ are the largest and the smallest, respectively.

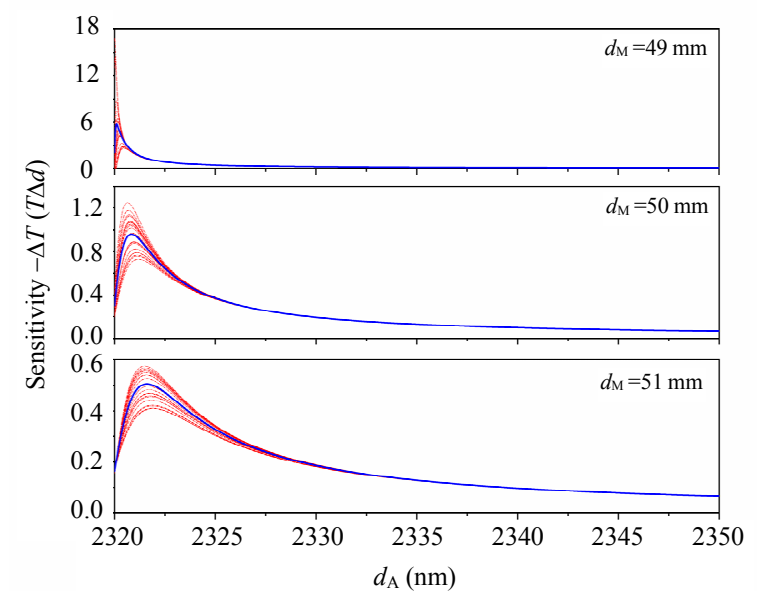

Fig. 8 Sensitivity versus $d_{\mathrm{A}}$ of three structures.

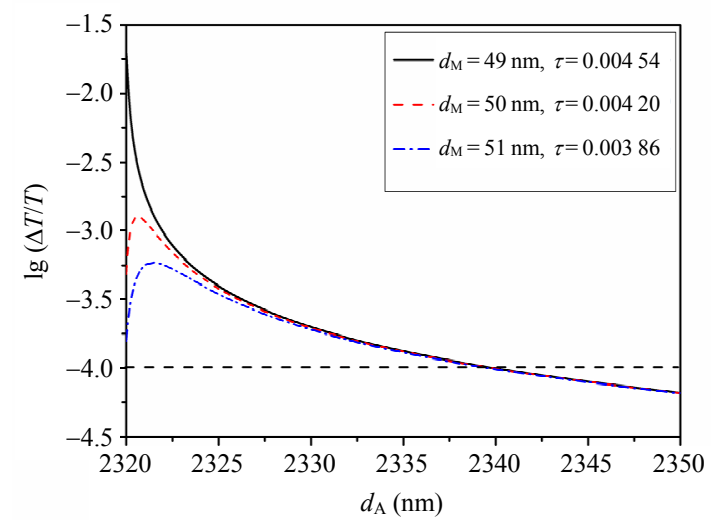

Fig. 9 Values of $\lg (\Delta T / T)$ corresponding to $\Delta d=$ $0.001 \mathrm{~nm}$ for three structures.

In processing real instrument, it is difficult to keep the exact balance of gain and loss in the two resonators. Thus, it is necessary to study the effect of non-balanced structure on the sensor performance. Here $n_{\mathrm{L}}=n+\mathrm{i} \tau$ is kept invariant and $n_{G}$ is made as $n_{\mathrm{G}}=n-\mathrm{i}(\tau+\Delta \tau) \quad(\Delta \tau$ is the non-balanced perturbation). The values of $\Delta T / T$ for the three structures with different $\Delta \tau$ are calculated. The results are shown in Fig. 11. It is clear that the values of $\Delta T / T$ for $d_{\mathrm{M}}=49 \mathrm{~nm}$ and $d_{\mathrm{M}}=51 \mathrm{~nm}$ are the largest and the smallest, respectively. Therefore, the sensitivity and the fault tolerance capability are contradictory. The structure with $d_{\mathrm{M}}=49 \mathrm{~nm}$ has the highest sensitivity but the lowest fault-tolerant capability. A trade-off is needed between the sensitivity and the fault-tolerant capability in real applications.

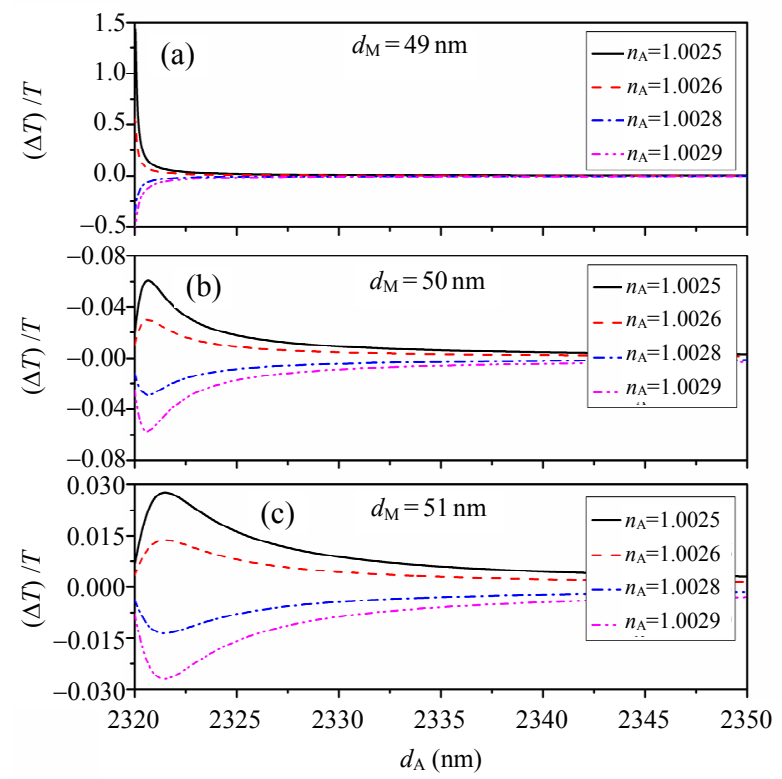

Fig. 10 Relative difference of the transmittances versus $d_{\mathrm{A}}$ for different $n_{\mathrm{A}}$ : (a) $d_{\mathrm{M}}=49 \mathrm{~nm}$, (b) $d_{\mathrm{M}}=50 \mathrm{~nm}$, and (c) $d_{\mathrm{M}}=$ $51 \mathrm{~nm}$.
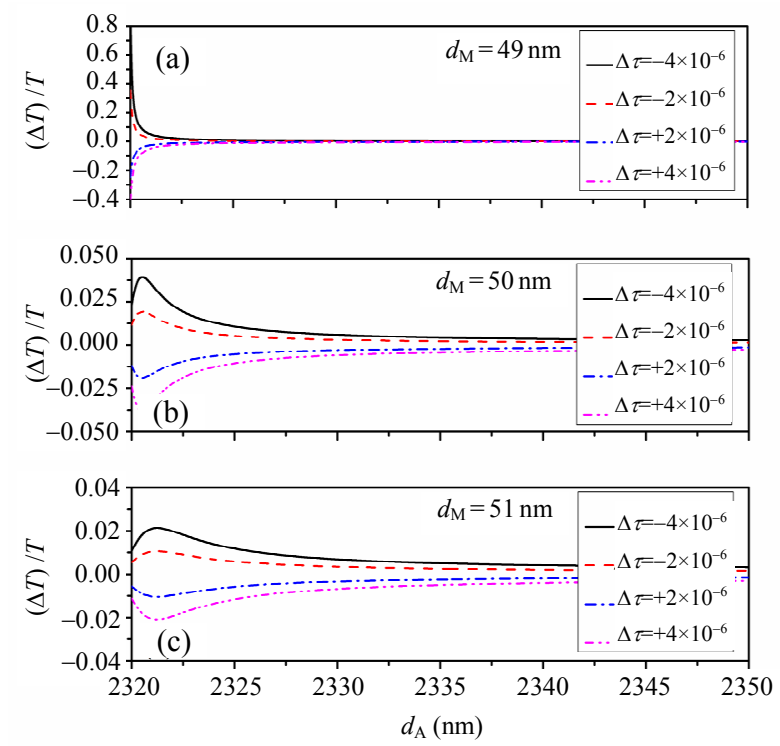

Fig. 11 Relative difference of the transmittances versus $d_{\mathrm{A}}$ for different $\Delta \tau$ : (a) $d_{\mathrm{M}}=49 \mathrm{~nm}$, (b) $d_{\mathrm{M}}=50 \mathrm{~nm}$, and (c) $d_{\mathrm{M}}=$ $51 \mathrm{~nm}$. 


\section{Conclusions}

In this paper, the coupling of two resonators with balanced gain and loss through an air layer has been studied. The pole effect can make the structure system become the displacement sensor. The sensor takes great advantages over the traditional displacement sensors because of its ultra-high resolution of $0.001 \mathrm{~nm}$, high sensitivity within one nanometer, and simple structure. It has avoided the large processing cost of the sensors based on optical-gratings and laser interferometers. The only limitation is the difficulty to realize the balanced gain and loss resonators. But the difficulty has been overcome in recent experiment studies. This study will open a new door to design sensors by PT-symmetry optics.

\section{Appendix}

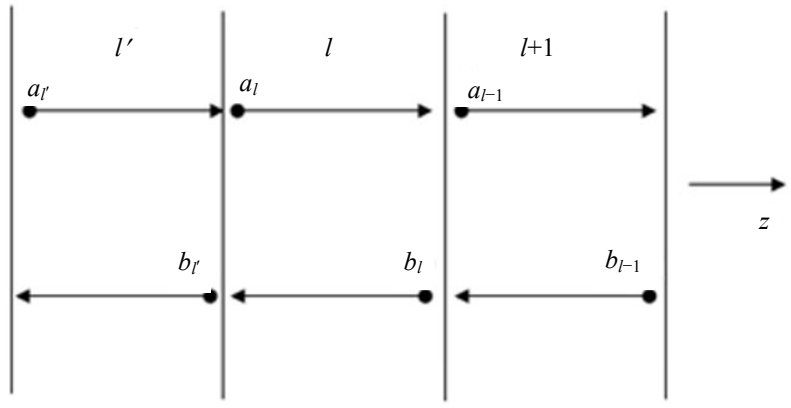

Fig. 12 Schematic of scatter matrix elements.

This part presents the deduction of scatter matrix. In Fig. 12, $a_{l}$ and $b_{l}$ are the amplitudes of forward and backward waves in an arbitrary layer $l$, respectively. For the two neighboring layers $l^{\prime}$ and $l$, the four wave amplitudes $a_{l}, b_{l}, a_{l^{\prime}}$, and $b_{l^{\prime}}$ are related by a scatter matrix $\mathbf{S}\left(l^{\prime}, l\right)$ as follows:

$$
\left[\begin{array}{l}
a_{l} \\
b_{l^{\prime}}
\end{array}\right]=\mathbf{S}\left(l^{\prime}, l\right)\left[\begin{array}{l}
a_{l^{\prime}} \\
b_{l}
\end{array}\right] \text {. }
$$

By that analogy, the four wave amplitudes in layers $l^{\prime}$ and $l+1$ can be written as follows:

$$
\left[\begin{array}{c}
a_{l+1} \\
b_{l^{\prime}}
\end{array}\right]=\mathbf{S}\left(l^{\prime}, l+1\right)\left[\begin{array}{c}
a_{l^{\prime}} \\
b_{l+1}
\end{array}\right] .
$$

Defining $\quad \hat{f}_{1}=\hat{f}\left(d_{l}\right)=\mathrm{e}^{\mathrm{i} q_{l} d_{l}} \quad\left(q_{l} \quad\right.$ is the $\quad z$ component of wave vector in layer $l$ ) leads to

$$
\left[\begin{array}{c}
\hat{f}_{l} a_{l} \\
b_{l}
\end{array}\right]=\mathbf{I}(l, l+1)\left[\begin{array}{c}
a_{l+1} \\
\hat{f}_{l+1} b_{l+1}
\end{array}\right]
$$

where $\mathbf{I}\left(l^{\prime}, l\right)=\frac{1}{2}\left[\begin{array}{cc}1+\frac{1}{k} \frac{q_{l}}{q_{l^{\prime}}} & 1-\frac{1}{k} \frac{q_{l}}{q_{l^{\prime}}} \\ 1-\frac{1}{k} \frac{q_{l}}{q_{l^{\prime}}} & 1+\frac{1}{k} \frac{q_{l}}{q_{l^{\prime}}}\end{array}\right],(k=1$ and $k=\left(\frac{n_{l}}{n_{l^{\prime}}}\right)^{2}$ correspond to TE and TM waves, respectively) is the matrix determining the electromagnetic field transition on the interface. Through some deduction, one can obtain the four elements of $\mathbf{S}\left(l^{\prime}, l+1\right)$ from $\mathbf{S}\left(l^{\prime}, l\right)$ as follows:

$$
\begin{gathered}
S_{11}\left(l^{\prime}, l+1\right)=\left[I_{11}-\hat{f}_{l} S_{12}\left(l^{\prime}, l\right) I_{21}\right]^{-1} \hat{f}_{l} S_{11}\left(l^{\prime}, l\right) \\
S_{12}\left(l^{\prime}, l+1\right)=\left[I_{11}-\hat{f}_{l} S_{12}\left(l^{\prime}, l\right) I_{21}\right]^{-1} \times \\
{\left[\hat{f}_{l} \hat{f}_{l+1} S_{12}\left(l^{\prime}, l\right) I_{22}-I_{12} \hat{f}_{l+1}\right]} \\
S_{21}\left(l^{\prime}, l+1\right)=S_{21}\left(l^{\prime}, l\right)+S_{22}\left(l^{\prime}, l\right) I_{21} S_{11}\left(l^{\prime}, l+1\right) \\
S_{22}\left(l^{\prime}, l+1\right)=S_{12}\left(l^{\prime}, l+1\right)+I_{22} \hat{f}_{l+1} .
\end{gathered}
$$

For $N$-layer structure, the initial matrix is $\mathbf{S}(0,0)=\left[\begin{array}{ll}1 & 0 \\ 0 & 1\end{array}\right]$. Equations (10)-(13) will lead to the total scatter matrix $\mathbf{S}(0, N)$.

Open Access This article is distributed under the terms of the Creative Commons Attribution 4.0 International License (http://creativecommons.org/licenses/by/4.0/), which permits unrestricted use, distribution, and reproduction in any medium, provided you give appropriate credit to the original author(s) and the source, provide a link to the Creative Commons license, and indicate if changes were made.

\section{References}

[1] H. I. Rasool, P. R. Wilkinson, A. Z. Steig, and J. K. Gimzewski, "A low noise all-fiber interferometer for high resolution frequency modulated atomic force microscopy," The Review of Scientific Instruments, 2010, 81(2): 023703-1-023703-10.

[2] B. K. Nowakowski, D. T. Smith, and S. T. Smith, "Highly compact fiber Fabry-Perot interferometer: a new instrument design," The Review of Scientific 
Instruments, 2016, 87(11): 115102-1-115102-8.

[3] F. G. Cervantes, L. Kumanchik, J. Pratt, and J. M. Taylor, "High sensitivity optomechanical reference accelerometer over $10 \mathrm{kHz}, "$ Applied Physics Letters, 2014, 104: 221111-1-221111-5.

[4] K. Peng, X. K. Liu, Z. R. Chen, Z. C. Yu, and H. J. $\mathrm{Pu}$, "Sensing mechanism and error analysis of a capacitive long-range displacement nanometer sensor based on time grating," IEEE Sensors Journal, 2017, 17(6): 1596-1607.

[5] C. C. Wu, C. H. Liao, Y. Z. Chen, and J. S. Yang, "Common-path laser encoder with Littrow configuration," Sensors \& Actuators A: Physical, 2013, 193(4): 69-78.

[6] X. L. Zhou and Q. X. Yu, "Wide-range displacement sensor based on fiber-optic Fabry-Perot interferometer for subnanometer measurement," IEEE Sensors Journal, 2011, 11(7): 1602-1606.

[7] C. Yang and S. O. Oyadiji, "Development of two-layer multiple transmitter fibre optic bundle displacement sensor and application in structural health monitoring," Sensors and Actuators A: Physical, 2016, 244(15): 1-14.

[8] Y. Yang, D. Tian, K. Chen, X. L. Zhou, Z. F. Gong, and Q. X. Yu, "A fiber-optic displacement sensor using the spectral demodulation method," Journal of Lightwave Technology, 2018, 36(17): 3666-3671.

[9] T. P. Dao, N. L. Ho, T. T. Nguyen, H. G. Le, P. T. Thang, H. T. Pham, et al., "Analysis and optimization of a micro-displacement sensor for compliant microgripper," Microsystem Technologies, 2017, 23(12): 5375-5395.

[10] T. P. Dao and S. C. Huang, "Design and analysis of a compliant micro-positioning platform with embedded strain gauges and viscoelastic damper," Microsystem Technologies, 2016, 23(2): 441-456.

[11] C. Yang and S. O. Oyadiji, "Theoretical and experimental study of self-reference intensity-modulated plastic fibre optic linear array displacement sensor," Sensors \& Actuators A: Physical, 2015, 222: 67-79.

[12] W. Gao, S. W. Kim, H. Bosse, H. Haitjema, Y. L. Chen, X. D. Lu, et al., "Measurement technologies for precision positioning," CIRP Annals, 2015, 64(2): 773-796.

[13] A. J. Fleming, "A review of nanometer resolution position sensors-operation and performance," Sensors and Actuators A: Physical, 2013, 190: 106-126.
[14] T. Grotjohann, I. Testa, M. Leutenegger, H. Leutenegger, H. Bock, N. T. Urban et al., "Diffraction-unlimited all-optical imaging and writing with a photochromic GFP," Nature, 2011, 478: 204-208.

[15] J. Baxter, "Super-resolution imaging: beyond the limit," Nature Photonics, 2012, 6(6): 273-275.

[16] G. Doyen, D. Drakova, V. Mujica, and M. Scheffler, "Theory of the scanning tunneling microscope," Physica Status Solidi, 1992, 131: 107-108.

[17] S. T. Souza, E. J. S. Fonseca, C. Jacinto, N. G. C. Astrath, T. P. Rodrigues, and L. C. Malacarne, "Direct measurement of photo-induced nanoscale surface displacement in solids using atomic force microscopy," Optical Materials, 2015, 48: 71-74.

[18] L. Feng, R. E. Ganainy, and L. Ge, "Non-hermitian photonics based on parity-time symmetry," Nature Photonics, 2017, 11: 752-762.

[19]Z. J. Wong, Y. L. Xu, J. Kim, K. O'Brien, Y. Wang, L. Feng, et al., "Lasing and anti-lasing in a single cavity," Nature Photonics, 2016, 10(12): 796-801.

[20] H. Hodaei, A. U. Hassan, S. Wittek, H. G. Gracia, R. E. Ganainy, D. N. Christodoulides, et al., "Enhanced sensitivity at higher-order exceptional points," Nature, 2017, 548: 187-191.

[21] P. Y. Chen, M. Sakhdari, M. Hajizadegan, Q. Cui, M. M. C. Cheng, R. E. Ganainy, et al., "Generalized parity-time symmetry condition for enhanced sensor telemetry," Nature Electronics, 2018, 1: 297-304.

[22]L. Chang, X. S. Jiang, S. Y. Hua, C. Yang, J. M. Wen, L. Jiang, et al., "Parity-time symmetry and variable optical isolation in active-passive-coupled microresonators," Nature Photonics, 2014, 8: 524-529.

[23] Y. D. Chong, L. Ge, and A. D. Stone, "PT-symmetry breaking and laser-absorber modes in optical scattering systems," Physical Review Letters, 2011, 106: 093902-1-093902-4.

[24] L. Ge, Y. D. Chong, and A. D. Stone, "Conservation relations and anisotropic transmission resonances in one-dimensional PT-symmetric photonic heterostructures," Physical Review A, 2012, 85: 023802-1-023802-10.

[25] W. L. Barnes, "Surface plasmon-polariton length scales: a route to sub-wavelength optics," Journal of Optics A: Pure and Applied Optics, 2006, 8(4): S87-S93.

[26] P. Yeh, Optical waves in layered media. New York, USA: John Wiley \& Sons, 2005: 1-416. 